\title{
Quantitative Analysis of Microperfusion in Contrast- Induced Nephropathy Using Contrast-Enhanced Ultrasound: An Animal Study
}

\author{
Nieun $\mathrm{Se}^{1}$, Hyewon $\mathrm{Oh}^{1}$, Hyung Jung $0 \mathrm{~h}^{2}$, Yong Eun Chung ${ }^{1}$ \\ ${ }^{1}$ Department of Radiology, Severance Hospital, Yonsei University College of Medicine, Seoul, Korea; ${ }^{2}$ Department of Nephrology, Sheikh Khalifa \\ Specialty Hospital, Ras AlKhaimah, UAE
}

Objective: To investigate imaging biomarkers of microperfusion in contrast-induced nephropathy (CIN) using contrastenhanced ultrasound (CEUS).

Materials and Methods: The CIN model was fabricated by administering indomethacin $(10 \mathrm{mg} / \mathrm{kg}), \mathrm{L}-\mathrm{NAME}(15 \mathrm{mg} / \mathrm{kg})$, and iopamidol $(10 \mathrm{~mL} / \mathrm{kg})$ to Sprague-Dawley rats. After 24 hours, CEUS was performed on CIN $(n=6)$ and control $(n=6)$ rats with sulphur hexafluoride microbubbles (SonoVue). From time-intensity curves obtained from the kidney arriving time $(A T)$, acceleration time $(A C)$, time to peak (TTP), and peak enhancement (PE) were measured and compared between the groups. After CEUS, the rats were sacrificed, and cell apoptosis markers were evaluated to confirm the development of CIN. Results: Among CEUS parameters, AT $(7.8 \pm 1.6$ vs. $4.2 \pm 0.5 \mathrm{~s}, p=0.002)$, AC $(4.7 \pm 1.4$ vs. $2.0 \pm 0.4 \mathrm{~s}, p=0.002)$, and TTP $(12.5 \pm 2.9$ vs. $6.2 \pm 0.6 \mathrm{~s}, p=0.002)$ were significantly prolonged in the CIN group compared to controls. PE was significantly higher in the control group than in the CIN group (17.1 \pm 1.9 vs. $12.2 \pm 2.0 \mathrm{~dB}, p=0.004)$. In kidney tissue, mRNA and protein levels of the apoptotic makers were significantly higher in the CIN group than in the control group ( $p=$ 0.003 and $p=0.002$ ).

Conclusion: CEUS parameters can be used as imaging biomarkers for microperfusion in CIN. In rats with CIN, AT, AC, and TTP were significantly prolonged, while PE was significantly lower compared to controls.

Keywords: Contrast media; Acute kidney injury; Perfusion; Contrast-enhanced ultrasonography; Animal experimentation

\section{INTRODUCTION}

Contrast-induced nephropathy (CIN) is the acute impairment of renal function caused by the injection of iodinated contrast agents [1,2]. It is the major cause of iatrogenic renal failure during hospitalization. In most patients with CIN, there is a mild and transient decrease

Received: May 8, 2020 Revised: August 10, 2020

Accepted: September 17, 2020

This study was supported by a faculty research grant of Yonsei University College of Medicine (6-2017-0070).

Corresponding author: Yong Eun Chung, MD, PhD, Department of Radiology, Severance Hospital, Yonsei University College of Medicine, 50-1 Yonsei-ro, Seodaemun-gu, Seoul 03722, Korea.

- E-mail:yelv@yuhs.ac

This is an Open Access article distributed under the terms of the Creative Commons Attribution Non-Commercial License (https://creativecommons.org/licenses/by-nc/4.0) which permits unrestricted non-commercial use, distribution, and reproduction in any medium, provided the original work is properly cited. in renal function. However, although rare, CIN can cause serious chronic renal dysfunction requiring renal replacement therapy [3-7]. Moreover, it is known to increase the duration of hospital stay and long-term morbidity and mortality, particularly in patients with pre-existing chronic renal dysfunction or diabetes [3-6]. Therefore, there is growing interest in finding ways to reduce CIN by understanding its pathophysiology and developing relevant biomarkers.

Although the pathophysiology of CIN is complex and has not been clearly understood, hemodynamic changes, including renal vasoconstriction, are thought to be the primary factors contributing to CIN $[8,9]$. Prolonged renal vasoconstriction results in decreased renal blood flow and glomerular filtration rate $[1,8]$. Considering these hemodynamic alterations, several studies have shown that the magnetic resonance imaging (MRI) techniques that reflect tissue perfusion, such as intravoxel incoherent 
motion (IVIM) or arterial spin labeling (ASL), might be useful for understanding the pathophysiology of CIN $[10,11]$. However, the clinical application of functional MRI, including IVIM, is limited because of the lack of standardized sequences, post-processing software, and limited accessibility.

Contrast-enhanced ultrasound (CEUS) enables continuous real-time imaging of vascular perfusion [12-14]. Ultrasound contrast agents are composed of gas microbubbles surrounded by a shell $[13,15]$. As the rheology and size of these contrast agents are similar to those of red blood cells, they can function as red blood cell tracers, allowing for perfusion analyses in various organs $[16,17]$. Moreover, CEUS has the advantages of wide accessibility, renal safety of contrast agents, high tolerance, and lack of radiation exposure [18]. The quantification of renal perfusion using CEUS could be valuable for understanding the pathophysiology underlying renal disease processes, including CIN, because decreased renal perfusion usually precedes the impairment of renal function [19]. However, to our knowledge, there have been no reports using CEUS to evaluate CIN. Therefore, this study was aimed at investigating potential imaging biomarkers of microperfusion in CIN using CEUS in an animal model.

\section{MATERIALS AND METHODS}

\section{Animal Experiments}

Twelve male Sprague-Dawley rats (OrientBio), approximately 1-2 months old and weighing 220-250 g, that had been housed in an animal laboratory were used. All experimental procedures were approved by the Department of Laboratory Animal Resources and Ethical Committee for animal studies. All animals were housed in a plastic cage while being allowed access to food and water and maintained in a 12-hours light and 12-hours dark cycle with $50 \pm 100 \%$ humidity and $22^{\circ} \mathrm{C} \pm 2^{\circ} \mathrm{C}$ temperature before the experiment. The CIN model was developed after 16-24-hours water restriction, followed by intraperitoneal injections of $10 \mathrm{mg} / \mathrm{kg}$ indomethacin (Sigma-Aldrich) and $15 \mathrm{mg} / \mathrm{kg}$ L-NAME (Cayman Chemical). After 15 minutes, $10 \mathrm{~mL} / \mathrm{kg}$ iopamidol (Pamiray 370, Dongkuk Pharm.) was injected intravenously. In addition to contrast agents, pretreatment with indomethacin and L-NAME, which inhibit vasodilatation, has been recommended to develop a reliable CIN rat model $[20,21]$. Rats were housed again with free access to water. After 24 hours, CEUS was performed.
Finally, rats were euthanized, and blood and kidney tissues were harvested. To confirm the development of CIN, serum creatinine and blood urea nitrogen were measured using the Cobas C502 (Roche) chemistry analyzer.

\section{Contrast-Enhanced Ultrasound}

CIN $(n=6)$ and control $(n=6)$ rats underwent CEUS using an ultrasound scanner (Logiq E9, GE Healthcare) with a $12.5-\mathrm{MHz}$ linear probe. During US examinations, rats were anesthetized with a mixture of tiletamine-zolazepam (Zoletil 50, Virbac Laboratories; $30 \mathrm{mg} / \mathrm{kg}$ ) and xylazine (Rompun, Bayer Korea; $10 \mathrm{mg} / \mathrm{kg}$ ), administered as an intraperitoneal injection. Before the contrast injection, native B-mode imaging was performed for both kidneys to select the kidney with a better sonic window for CEUS. Subsequently, rats were injected with $0.6 \mathrm{~mL}$ of sulphur hexafluoride microbubbles (SonoVue; Bracco SpA) in the tail vein using an infusion pump (Terufusion, Terumo) at a rate of $5 \mathrm{~mL} / \mathrm{min}$. Renal CEUS was performed with a low mechanical index (0.08), and CEUS scans were acquired for 5 minutes after the contrast injection (Fig. 1). Scans were analyzed using software (QLAB, Philips Healthcare) dedicated for CEUS analyses. Two board-certified abdominal radiologists independently performed the CEUS analysis to calculate the interobserver agreement. After more than 1 month, a radiologist analyzed the CEUS scans again to calculate the intra-observer agreement. Three regions of interest (ROIs) were manually drawn in the kidney to include the cortex and outer medulla, and a time-intensity curve (TIC) was obtained (Fig. 1). TIC parameters, including the arriving time (AT), acceleration time $(\mathrm{AC})$, time to peak (TTP), and peak enhancement (PE), were measured, and the mean value of the three ROIs was compared between the two groups. Each parameter was defined as follows (Fig. 2): AT, interval from the contrast injection to the beginning of enhancement; $A C$, interval from the beginning of enhancement to PE; TTP, interval from the contrast injection to $\mathrm{PE}$; and $\mathrm{PE}$, maximal signal intensity measured in the selected ROI.

\section{Histological Examination and Immunohistochemistry of the Kidneys}

Kidney specimens were fixed in $10 \%$ neutral buffered formalin (Biosesang) for 24 hours, and paraffin blocks were made according to the previously published standard methods [22]. Paraffin blocks were then cut into $4-\mu \mathrm{m}$ sections, and paraffin-embedded tissues were 

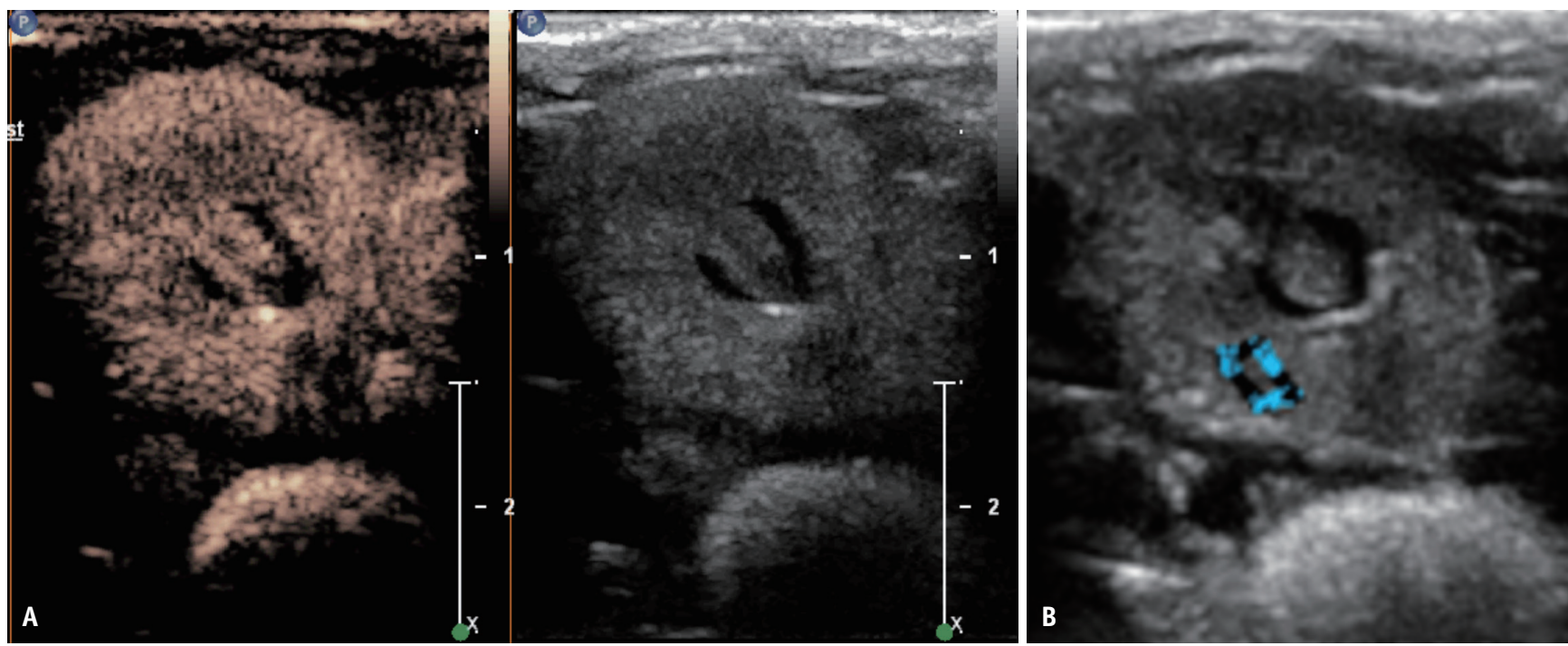

Fig. 1. Renal contrast-enhanced ultrasound in a rat model.

A. B-mode imaging (right) and contrast-enhanced imaging (left) after the contrast injection. B. A region of interest (blue line) was placed in the kidney to include the cortex and outer medulla, and a time-intensity curve was obtained.

de-paraffinized by changes in xylene and rehydrated in decreasing concentrations of ethanol $(100 \%, 95 \%$, $80 \%$, and $70 \%$ ). The sections were then stained with hematoxylin and eosin (Gill III, Merck) using an automatic strainer (Leica). For the immunohistochemistry staining of cleaved caspase-3, paraffin block sections were immersed in methanol (Duksan) with $0.3 \% \mathrm{H}_{2} \mathrm{O}_{2}$ for 10 minutes at room temperature to quench endogenous peroxidase activity. Subsequently, they were washed twice with Trisbuffered saline and blocked with $5 \%$ bovine serum albumin in phosphate-buffered saline. After adding the cleaved caspase-3 antibody (1:1000 dilution, \#9664, Cell Signaling Technologies) as the primary antibody for 1 hour at room temperature, they were washed twice with Tris-buffered saline and supplied with biotinylated goat-anti-rabbit IgGHRP (1:10000 dilution, \#K4003 from DAK0, Glostrup) as the secondary antibody. The stained slides were observed with fluorescent microscopy (IX71/DP71, Olympus).

\section{Transmission Electron Microscopy}

The kidney tissue morphology was examined with transmission electron microscopy (TEM). The tissues were fixed overnight in $0.1 \mathrm{M}$ phosphate buffer ( $\mathrm{pH} 7.4$ ) containing $2 \%$ glutaraldehyde, $2 \%$ paraformaldehyde, and $0.5 \% \mathrm{CaCl}_{2}$. The samples were washed twice with $0.1 \mathrm{M}$ phosphate buffer for 30 minutes and then fixed for 2 hours with $1 \% \mathrm{O}_{\mathrm{s}} \mathrm{O}_{4}$ dissolved in $0.1 \mathrm{M}$ phosphate buffer, followed by dehydration through a series of ethanol concentrations (50-100\% with increments of $10 \%$ ), allocating 10 minutes

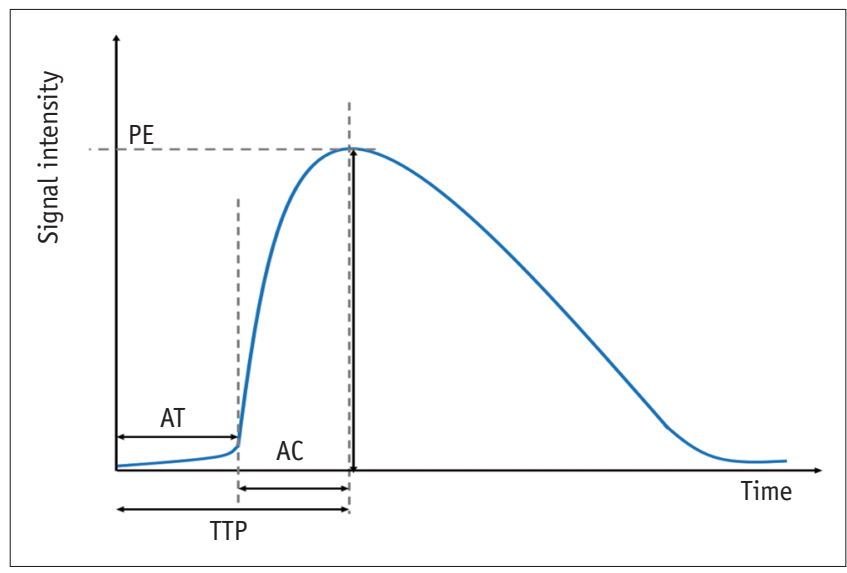

Fig. 2. Diagram of parameters according to the time-intensity curve. $A C=$ acceleration time, $A T=$ arriving time, $P E=$ peak enhancement, TTP $=$ time to peak

for each concentration. Specimens were embedded with the Poly/Bed812 kit (Polysciences, Inc.) and polymerized at $65^{\circ} \mathrm{C}$ in an electron micro-oven (TD-700, DOSAKA) for 24 hours. The block was cut using an ultramicrotome (LEICA EM UC-7, Leica Microsystem) and observed with TEM (JEM1011, JEOL) at an acceleration voltage of $80 \mathrm{kV}$ [23].

\section{RNA Extraction and Quantitative Real-Time Polymerase Chain Reaction}

Total RNA was isolated with a commercial kit (Hybrid-R kit 305-101, GeneAll Biotechnology), and $1 \mu \mathrm{g}$ of total RNA was reverse transcribed with the amfiRivert CDNA synthesis kit (GenDEPOT) according to the manufacturer's instructions. The expression levels of $\mathrm{Bax}, \mathrm{Bcl}-2$, neutrophil gelatinase- 
associated lipocalin (NGAL), and interleukin 18 (IL-18) were determined with real-time polymerase chain reaction (RT-PCR) using the SYBR-Green reagent (GenDEPOT) and normalized to $\beta$-actin. Bax and $\mathrm{Bcl}-2$ are apoptosis-related genes, while NGAL and IL-18 are biomarkers of acute kidney injury $[24,25]$. For Bax/Bcl2 expression levels, the relative ratio (Bax/ $\beta$-actin) $/(\mathrm{Bcl} 2 / \beta$-actin) was calculated by dividing the separately calculated ratio (Bax/ $\beta$-actin) by (Bcl2/ $\beta$-actin). All PCR reactions were conducted in duplicate, and threshold cycle $(\mathrm{Ct})$ values were analyzed with the $2^{-\Delta \Delta \mathrm{Ct}}$ methods.

\section{Western Blot Analysis}

The Western blot analysis was performed using the previously described method [23]. In summary, kidney tissue was homogenized and lysed in a pro-prep extraction solution (iNtRON Biotechnologist), followed by protein quantitation with the Bradford method. Lysates were fractioned on $15 \%$ sodium dodecyl sulfate-polyacrylamide gels and transferred to polyvinylidene difluoride membranes. The membrane was incubated overnight at $4^{\circ} \mathrm{C}$ with primary antibodies against Bax 1:1000 dilution, \#2772 (Cell Signaling Technologies), Bcl-2 1:1000 dilution, \#ab59348 (Abcam), and $\beta$-actin 1:10000 dilution, \#LFPA0207 (AbFrontier), used as the loading controls. After the membrane was washed thrice in $1 \mathrm{X}$ Tris-Buffered Saline with Tween (TBS-T) for 15 minutes each, it was incubated with the HRP-conjugated secondary antibodies (goat anti-rabbit IgG-HRP, 1:10000 dilution, \#SA002-500 from GenDepot) for 1 hour at room temperature. Subsequently, the membrane was washed thrice in $1 \mathrm{X}$ TBS-T for 15 minutes again. The blotted membrane was visualized with enhanced chemiluminescence reagents (GenDEPOT) and exposed to an X-ray film. The results were normalized to the $\beta$-actin loading control, and band density was measured using Image $\mathrm{J}$ software (NIH; online at https://imagej.nih.gov/ij/).

\section{Statistical Analysis}

All results are expressed as mean \pm standard deviation. The mean values of the rat body weight, kidney function analysis, CEUS data, and histopathological parameters were compared between the control and CIN groups using the non-parametric Mann-Whitney $U$ test. The inter- and intraobserver agreements for CEUS parameters were calculated using the intraclass correlation coefficients (ICCS). ICC values $\geq 0.75,0.60-0.74,0.40-0.59$, and $<0.40$ represent excellent, good, fair, and poor agreements, respectively. Correlations between serum creatinine and CEUS parameters were evaluated by Spearman's rank correlation analysis. Statistical analyses were performed using Prism 8.3.0 (Graphpad) and SPSS v25.0 software (IBM Corp.). All $p$ values were two-sided, and $p$ values $<0.05$ were considered statistically significant.

\section{RESULTS}

\section{Kidney Function Analysis}

There was no statistical difference in the rat body weight between the control $(292.9 \pm 13.4 \mathrm{~g})$ and CIN $(295.4 \pm$ $24.2 \mathrm{~g} ; p=0.818)$ groups. The levels of blood urea nitrogen $(13.5 \pm 2.0$ vs. $171.2 \pm 45.4 \mathrm{mg} / \mathrm{dL}, p=0.002)$ and creatinine $(0.3 \pm 0.0$ vs. $1.6 \pm 0.5 \mathrm{mg} / \mathrm{dL}, p=0.002)$ were higher in the CIN group than in the control group.

\section{CEUS Parameters}

Table 1 summarizes the parameters of renal perfusion, and Figure 3 shows the representative TICs of both groups. AT and AC were significantly prolonged in the CIN group than in the control group ( $7.8 \pm 1.6$ vs. $4.2 \pm 0.5 \mathrm{~s}, p=$ 0.002 for AT; and $4.7 \pm 1.4$ vs. $2.0 \pm 0.4 \mathrm{~s}, p=0.002$ for AC). TTP also significantly increased in the CIN group (12.5 $\pm 2.9 \mathrm{~s})$ than in the control group $(6.2 \pm 0.6 \mathrm{~s}, p=0.002)$. $P E$ was significantly higher in the control group than in the CIN group ( $17.1 \pm 1.9$ vs. $12.2 \pm 2.0 \mathrm{~dB}, p=0.004)$. For the CEUS analysis, the inter-observer agreement was good to excellent (ICC, 0.726-0.991), and intra-observer agreement was excellent (ICC, 0.902-0.995) (Table 2).

All CEUS parameters significantly correlated with serum creatinine levels. AT $(\rho=0.886, p<0.001)$, AC $(\rho=0.829$, $p=0.001)$, and $\operatorname{TTP}(\rho=0.850, p<0.001)$ positively correlated with serum creatinine, whereas PE negatively correlated with serum creatinine $(\rho=-0.722, p=0.008)$.

\section{Histological Morphology of the Kidneys}

Figure 4 shows the histological findings of the control and CIN groups. With the hematoxylin and eosin staining, there was no obvious change in the tubule and glomerulus

Table 1. Contrast-Enhanced Ultrasound Parameters

\begin{tabular}{lcrc}
\hline \multicolumn{1}{c}{ Parameters } & Control $(n=6)$ & CIN $(n=6)$ & \multicolumn{1}{c}{$P$} \\
\hline AT $(\mathrm{sec})$ & $4.2 \pm 0.5$ & $7.8 \pm 1.6$ & 0.002 \\
AC $(\mathrm{sec})$ & $2.0 \pm 0.4$ & $4.7 \pm 1.4$ & 0.002 \\
TTP $(\mathrm{sec})$ & $6.2 \pm 0.6$ & $12.5 \pm 2.9$ & 0.002 \\
PE $(\mathrm{dB})$ & $17.1 \pm 1.9$ & $12.2 \pm 2.0$ & 0.004 \\
\hline
\end{tabular}

$\mathrm{AC}=$ acceleration time, $\mathrm{AT}=$ arriving time, $\mathrm{CIN}=$ contrast-induced nephropathy, $\mathrm{PE}=$ peak enhancement, TTP = time to peak 


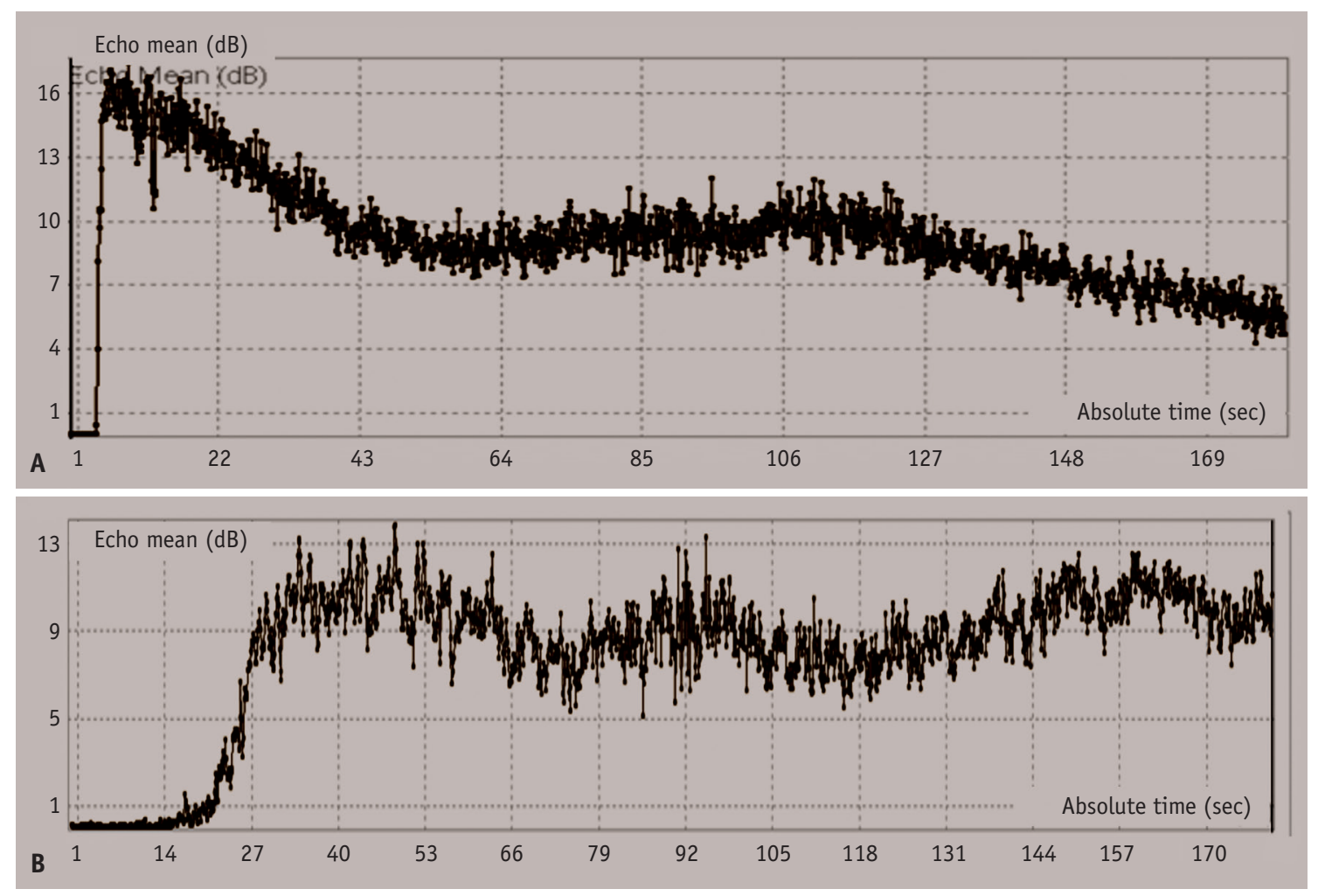

Fig. 3. TICs in control (A) and CIN models (B). TIC in the CIN model (B) shows prolonged arriving time, acceleration time, and time to peak compared to the TIC in the control model (A). Peak enhancement is slightly higher in the control model (A) than in the CIN model (B). CIN = contrast-induced nephropathy, TIC = time-intensity curve

Table 2. Inter-Observer and Intra-Observer Agreements for Contrast-Enhanced Ultrasound Analysis

\begin{tabular}{ccccc}
\hline Parameters & Inter-0bserver & $95 \%$ CI & Intra-0bserver & $95 \%$ CI \\
\hline AT $(\mathrm{sec})$ & 0.991 & $0.967-0.997$ & 0.995 & $0.983-0.999$ \\
AC (sec) & 0.772 & $0.208-0.934$ & 0.974 & $0.909-0.992$ \\
TTP (sec) & 0.955 & $0.843-0.987$ & 0.992 & $0.972-0.998$ \\
PE (dB) & 0.726 & $0.048-0.921$ & 0.902 & $0.659-0.972$ \\
\hline
\end{tabular}

$\mathrm{AC}=$ acceleration time, $\mathrm{AT}=$ arriving time, $\mathrm{CI}=$ confidence interval, $\mathrm{PE}=$ peak enhancement, $\mathrm{TTP}=$ time to peak

between the two groups (Fig. 4). With TEM, there were more cytoplasmic vacuoles and greater mitochondrial expansion in the tubular cells of the CIN group than in the control group (Fig. 4). Immunohistochemistry staining for cleaved caspase-3 showed apoptotic cells in the CIN group (Fig. 5).

\section{Gene Expression of Kidney Injury Markers}

To investigate the expression of kidney injury markers, the mRNA expression levels of NGAL and IL-18 were compared between the control and CIN groups with RT-PCR. The expression levels of NGAL and IL-18 significantly increased in the CIN group compared to controls $(p<0.001$ and $p=$ 0.003 , respectively) (Table 3, Fig. 6).

\section{Apoptosis-Related Gene Expression}

To determine the exposure effect of contrast agents on apoptosis, mRNA and protein expression levels of the apoptosis markers (Bax, $\mathrm{Bcl}-2)$ were assessed. The expression levels of mRNA and protein (Bax/Bcl-2) significantly increased in the CIN group compared to controls $(p=0.003$ and $p=0.002$, respectively) (Table 3, Fig. 7).

\section{DISCUSSION}

This study showed that hemodynamic alterations in CIN were evident on CEUS performed 24 hours after a contrast agent was injected. CEUS parameters, including AT, AC, and TTP, increased significantly in the CIN group compared to controls, whereas PE was significantly lower in the CIN group compared to controls. In addition, all CEUS parameters evaluated in our study revealed significant correlation with serum creatinine. This result indicates 

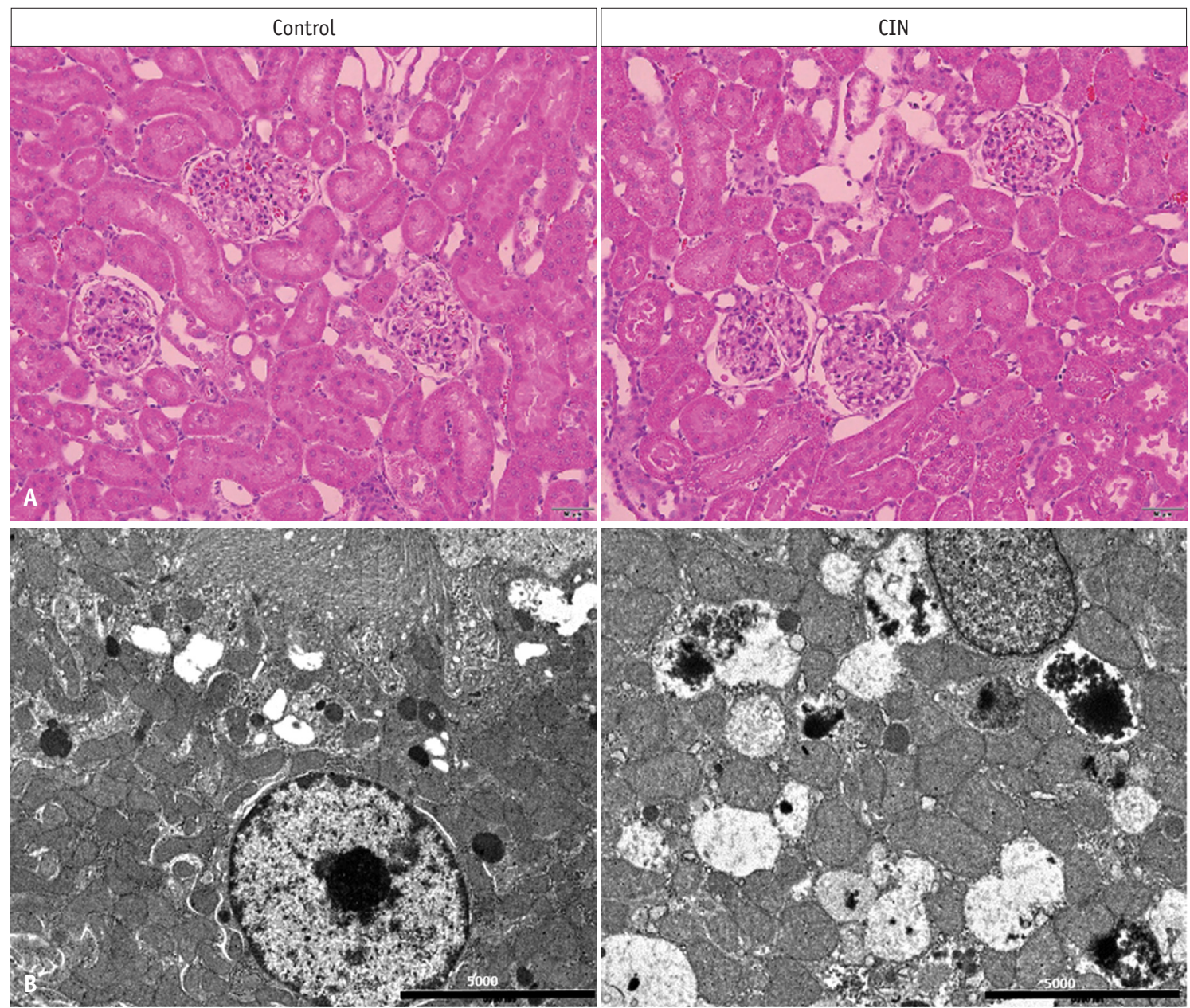

Fig. 4. Renal histological morphology in the control and CIN models.

A. Histological examination of kidney tissue with hematoxylin and eosin staining. There is no obvious change in tubular cells of the glomerulus in the CIN group compared to the control. Magnification $=x 20$, scale bar $=50 \mu \mathrm{m}$. B. Analysis of morphology changes in the kidney using transmission electron microscopy. In the CIN model, more vacuoles and greater mitochondrial expansion are observed. Magnification $=x 8000$, scale bar $=5000 \mathrm{~nm}$. CIN = contrast-induced nephropathy

that changes in CEUS parameters may reflect clinical renal dysfunction in CIN.

Although the pathophysiology of CIN is not well established, changes in vascular dynamics are a primary cause of acute tubular necrosis in CIN [8]. Prolonged renal vascular constriction results in increased renal vascular resistance, decreased renal blood flow, and decreased glomerular filtration rate, which make the outer medulla susceptible to necrosis $[1,26]$. Several imaging modalities have been used to evaluate renal vascular perfusion. CEUS is a competing technique that enables noninvasive and realtime assessment of perfusion $[13,27]$. Several studies have reported that CEUS can be used to evaluate renal perfusion in various diseases, including experimental renal hypoxia, microvascular perfusion dysfunction in diabetic GotoKakizaki rats, and renal transplant dysfunction $[14,16,28$ -
30]. Most studies on renal hypoxia or acute congestion showed increased TTP or mean transit time and decreased $\mathrm{PE}$, which indicate decreased renal flow velocity and blood volume, respectively $[16,28,30]$. Our study revealed similar changes in CEUS parameters as those previous studies.

Regarding other imaging modalities, functional MRI, such as IVIM or ASL, without administering contrast agents can also be used to evaluate renal perfusion $[10,11,31,32]$. A few studies have shown IVIM and ASL to be effective methods for monitoring the progression of CIN $[10,11]$. According to a previous study, renal blood flow measured with ASL significantly decreased in both the renal cortex and medulla in early stages of CIN (12-48 hours) [11]. Perfusion-related IVIM parameters, including perfusion fraction (f) and pseudo-diffusion coefficient $\left(D^{*}\right)$, also significantly decreased in the CIN model. In addition, there 

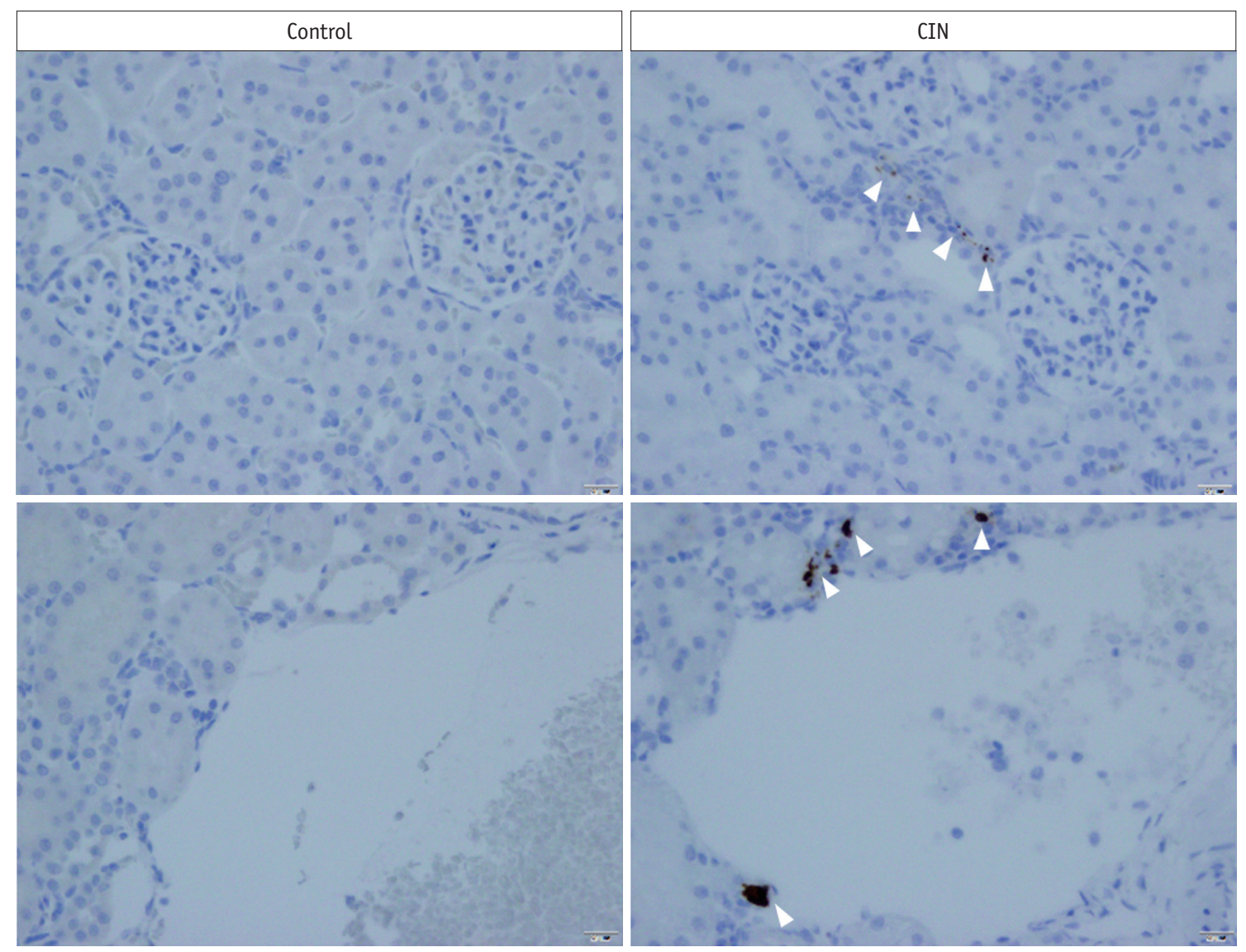

Fig. 5. Immunohistochemistry of cleaved caspase-3. Arrowheads indicate stained apoptotic cells in the CIN model. Magnification $=x 40$, scale bar $=20 \mu \mathrm{m}$. CIN $=$ contrast-induced nephropathy

Table 3. Gene Expression of Kidney Injury Markers and Apoptosis Markers

\begin{tabular}{lccr}
\hline & $\begin{array}{c}\text { Control } \\
(n=6)\end{array}$ & $\begin{array}{c}\text { CIN } \\
(n=6)\end{array}$ & $P$ \\
\hline mRNA expression level (AU) & & & \\
$\quad$ NGAL & 1 & $109.6 \pm 94.6$ & $<0.001$ \\
IL-18 & 1 & $4.5 \pm 3.6$ & 0.003 \\
Bax/Bcl2 & 1 & $1.8 \pm 0.8$ & 0.003 \\
Protein expression level (AU) & & & \\
$\quad$ Bax/Bcl2 & 1 & $10.6 \pm 4.9$ & 0.002 \\
\hline
\end{tabular}

$\mathrm{AU}=$ arbitrary unit, $\mathrm{CIN}=$ contrast-induced nephropathy, IL-18 = interleukin 18, NGAL = neutrophil gelatinase-associated lipocalin

was a strong correlation between perfusions measured with ASL and IVIM in the renal cortex. Interestingly, decreases in $D^{*}$ were observed before decreases in $f$ along time, which suggests that vasoconstriction might have occurred earlier than the decrease in fluid volume [11]. These MRI results are concordant with our results, although we did not investigate the serial changes in CEUS parameters in our study.

The histopathological examination revealed significantly

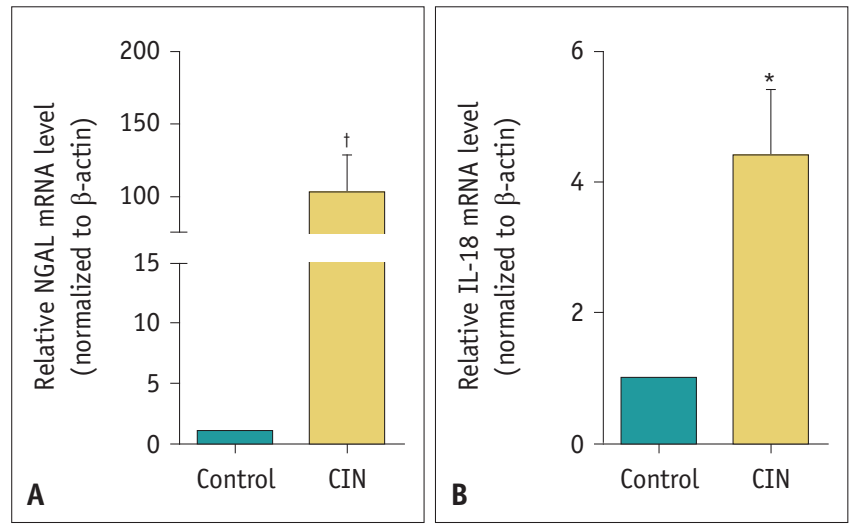

Fig. 6. mRNA expression levels of kidney injury markers (A: NGAL, B: IL-18 level). Statistical significance: ${ }^{*} p<0.01,{ }^{\dagger} p<$ 0.001 between the control and CIN groups. CIN $=$ contrast-induced nephropathy, IL-18 = interleukin 18, NGAL = neutrophil gelatinaseassociated lipocalin

increased kidney injury markers (NGAL, IL-18) and cell apoptosis markers (Bax/Bcl2, cleaved caspase-3) and correlated with morphological changes on TEM in our animal model. Serum creatinine, which is elevated for 1-3 days after the administration of contrast media, is 


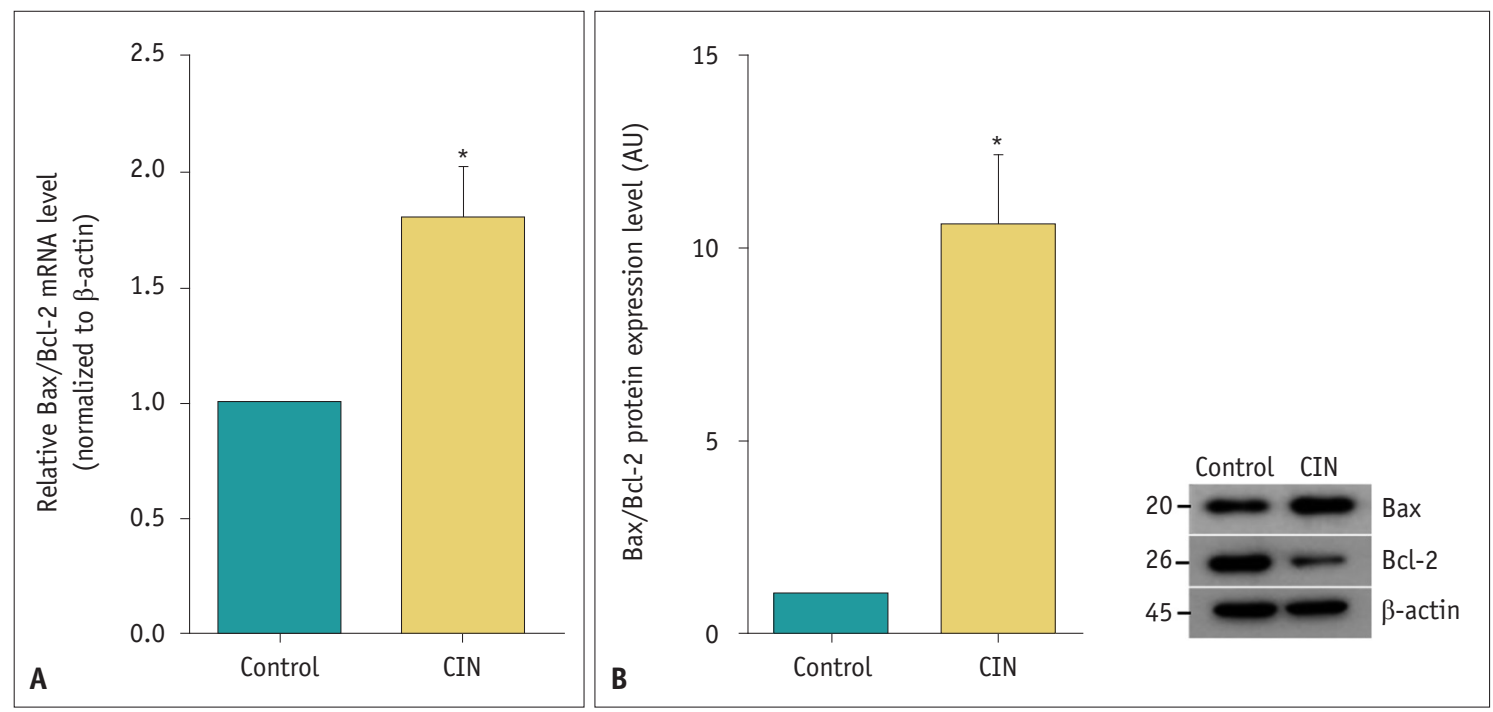

Fig. 7. mRNA (A) and protein (B) expression levels of the apoptotic markers (Bax/Bcl-2). Statistical significance: ${ }^{*} p<0.01$ between the control and CIN groups. AU = arbitrary unit, CIN = contrast-induced nephropathy

currently used as the diagnostic criterion for CIN. To date, early biomarkers of CIN, such as NGAL, cystatin C, kidney molecule-1, and IL-18, have been introduced, which are elevated only for a few hours after CIN develops [33,34]. In our study, the CIN group showed elevated serum creatinine and early biomarkers, including NGAL and IL-18, compared to the control group, which confirmed that the CIN model was well established. In addition, NGAL and IL-18 increased when ischemic injury occurred in the kidneys $[33,34]$. In our study, changes in CEUS parameters in the CIN group suggested delayed and decreased hemodynamic flow into the kidneys, which may have caused ischemic stress in the renal tissue and increased NGAL and IL-18 expression. However, as we did not evaluate a direct correlation between biomarkers and renal hemodynamic changes in this study, further studies are warranted.

This study had several limitations. First, the number of rats was small in the CIN and control groups. Second, the baseline CEUS parameters in CIN rats were not assessed before CIN development. Third, we performed the TIC analysis in the renal cortex and outer medulla. Although several previous studies on renal CEUS obtained TIC parameters from the cortex and medulla separately $[14,16,30]$, reliable ROI placement in the medulla was challenging because the medullary outline was difficult to define in most rats. Finally, the scan planes of CEUS and histopathological slides may not have exactly matched, and they were assumed to be representative of the whole kidney.

In conclusion, CEUS could be an effective imaging modality for evaluating CIN with the quantitative analysis of renal perfusion. In rats with CIN, AT, AC, and TTP were prolonged, whereas PE decreased, probably because of vasoconstriction, reflecting the hemodynamic changes in CIN. Further clinical studies with human subjects are required to use CEUS parameters as biomarkers for CIN pathophysiology and to develop prevention strategies for CIN.

\section{Conflicts of Interest}

The authors have no potential conflicts of interest to disclose.

\section{Author Contributions}

Conceptualization: Nieun Seo, Yong Eun Chung. Data curation: Nieun Seo, Hyewon Oh. Formal analysis: Nieun Seo, Hyewon Oh. Funding acquisition: Nieun Seo, Yong Eun Chung. Investigation: Nieun Seo, Yong Eun Chung, Hyung Jung Oh. Methodology: Nieun Seo, Yong Eun Chung. Project administration: Nieun Seo, Yong Eun Chung. Resources: Yong Eun Chung, Hyung Jung Oh. Supervision: Yong Eun Chung, Hyung Jung Oh. Visualization: Nieun Seo, Hyewon Oh. Writing - original draft: Nieun Seo, Hyewon Oh. Writing-review \& editing: Yong Eun Chung, Hyung Jung $0 h$.

\section{ORCID iDs}

Nieun Seo

https://orcid.org/0000-0001-8745-6454 
Hyewon 0 h

https://orcid.org/0000-0003-4985-8000

Hyung Jung $0 \mathrm{~h}$

https://orcid.org/0000-0002-4281-696X

Yong Eun Chung

https://orcid.org/0000-0003-0811-9578

\section{REFERENCES}

1. Murphy SW, Barrett BJ, Parfrey PS. Contrast nephropathy. J Am Soc Nephrol 2000;11:177-182

2. Parfrey PS, Griffiths SM, Barrett BJ, Paul MD, Genge M, Withers $\mathrm{J}$, et al. Contrast material-induced renal failure in patients with diabetes mellitus, renal insufficiency, or both. A prospective controlled study. N Engl J Med 1989;320:143-149

3. Abu Jawdeh BG, Kanso AA, Schelling JR. Evidencebased approach for prevention of radiocontrast-induced nephropathy. J Hosp Med 2009;4:500-506

4. Stacul F, Van der Molen AJ, Reimer P, Webb JA, Thomsen HS, Morcos SK, et al. Contrast induced nephropathy: updated ESUR Contrast Media Safety Committee guidelines. Eur Radiol 2011;21:2527-2541

5. Balemans CE, Reichert LJ, van Schelven BI, van den Brand JA, Wetzels JF. Epidemiology of contrast material-induced nephropathy in the era of hydration. Radiology 2012;263:706713

6. Weisbord SD, Mor MK, Resnick AL, Hartwig KC, Palevsky $\mathrm{PM}$, Fine MJ. Incidence and outcomes of contrast-induced AKI following computed tomography. Clin J Am Soc Nephrol 2008;3:1274-1281

7. Oh HJ, Kim S, Park JT, Kim SJ, Han SH, Yoo TH, et al. Baseline chloride levels are associated with the incidence of contrastassociated acute kidney injury. Sci Rep 2017;7:17431

8. Scharnweber T, Alhilali L, Fakhran S. Contrast-induced acute kidney injury: pathophysiology, manifestations, prevention, and management. Magn Reson Imaging Clin N Am 2017;25:743-753

9. McCullough PA, Choi JP, Feghali GA, Schussler JM, Stoler RM, Vallabahn RC, et al. Contrast-induced acute kidney injury. J Am Coll Cardiol 2016;68:1465-1473

10. Zhang B, Dong Y, Guo B, Chen W, Ouyang F, Lian Z, et al. Application of noninvasive functional imaging to monitor the progressive changes in kidney diffusion and perfusion in contrast-induced acute kidney injury rats at $3.0 \mathrm{~T}$. Abdom Radiol (NY) 2018;43:655-662

11. Liang L, Chen WB, Chan KW, Li YG, Zhang B, Liang CH, et al. Using intravoxel incoherent motion MR imaging to study the renal pathophysiological process of contrast-induced acute kidney injury in rats: comparison with conventional DWI and arterial spin labelling. Eur Radiol 2016;26:1597-1605

12. Granata A, Zanoli L, Insalaco M, Valentino M, Pavlica P, Di Nicolò PP, et al. Contrast-enhanced ultrasound (CEUS) in nephrology: has the time come for its widespread use? Clin Exp Nephrol 2015;19:606-615

13. Wang L, Mohan C. Contrast-enhanced ultrasound: a promising method for renal microvascular perfusion evaluation. J Transl Int Med 2016;4:104-108

14. Liu B, Liang F, Gu LP, Wang CQ, Li XH, Jiang YM, et al. Renal blood perfusion in GK rats using targeted contrast enhanced ultrasonography. Asian Pac J Trop Med 2015;8:668-673

15. Greis C. Technology overview: SonoVue (Bracco, Milan). Eur Radiol 2004;14 Suppl 8:P11-15

16. Brabrand K, De Lange C, Emblem KE, Reinholt FP, Saugstad OD, Stokke ES, et al. Contrast-enhanced ultrasound identifies reduced overall and regional renal perfusion during global hypoxia in piglets. Invest Radiol 2014;49:540-546

17. Chung YE, Kim KW. Contrast-enhanced ultrasonography: advance and current status in abdominal imaging. Ultrasonography 2015;34:3-18

18. McArthur C, Baxter GM. Current and potential renal applications of contrast-enhanced ultrasound. Clin Radiol 2012;67:909-922

19. Herget-Rosenthal S. Imaging techniques in the management of chronic kidney disease: current developments and future perspectives. Semin Nephrol 2011;31:283-290

20. Heyman SN, Brezis M, Epstein FH, Spokes K, Silva P, Rosen S. Early renal medullary hypoxic injury from radiocontrast and indomethacin. Kidney Int 1991;40:632-642

21. Chen YH, Fu YC, Wu MJ. Does resveratrol play a role in decreasing the inflammation associated with contrast induced nephropathy in rat model? J Clin Med 2019;8:147

22. Shi SR, Key ME, Kalra KL. Antigen retrieval in formalinfixed, paraffin-embedded tissues: an enhancement method for immunohistochemical staining based on microwave oven heating of tissue sections. J Histochem Cytochem 1991;39:741-748

23. Oh HJ, Oh H, Nam BY, You JS, Ryu DR, Kang SW, et al. The protective effect of klotho against contrast-associated acute kidney injury via the antioxidative effect. Am J Physiol Renal Physiol 2019;317:F881-F889

24. Tsigou E, Psallida V, Demponeras C, Boutzouka E, Baltopoulos $G$. Role of new biomarkers: functional and structural damage. Crit Care Res Pract 2013;2013:361078

25. Nguyen MT, Devarajan P. Biomarkers for the early detection of acute kidney injury. Pediatr Nephrol 2008;23:2151-2157

26. Detrenis S, Meschi M, Musini S, Savazzi G. Lights and shadows on the pathogenesis of contrast-induced nephropathy: state of the art. Nephrol Dial Transplant 2005;20:1542-1550

27. Greis C. Quantitative evaluation of microvascular blood flow by contrast-enhanced ultrasound (CEUS). Clin Hemorheol Microcirc 2011;49:137-149

28. Ma F, Yadav GP, Cang YQ, Dang YY, Wang CQ, Liu B, et al. Contrast-enhanced ultrasonography is a valid technique for the assessment of renal microvascular perfusion dysfunction in diabetic Goto-Kakizaki rats. Nephrology (Carlton) 2013;18:750-760 
29. Grabner A, Kentrup D, Pawelski H, Mühlmeister M, Biermann C, Edemir B, et al. Renal contrast-enhanced sonography findings in a model of acute cellular allograft rejection. $\mathrm{Am} \mathrm{J}$ Transplant 2016;16:1612-1619

30. Komuro K, Seo Y, Yamamoto M, Sai S, Ishizu T, Shimazu K, et al. Assessment of renal perfusion impairment in a rat model of acute renal congestion using contrast-enhanced ultrasonography. Heart Vessels 2018;33:434-440

31. Wentland AL, Artz NS, Fain SB, Grist TM, Djamali A, Sadowski EA. MR measures of renal perfusion, oxygen bioavailability and total renal blood flow in a porcine model: noninvasive regional assessment of renal function. Nephrol Dial Transplant 2012;27:128-135

32. Zhou HY, Chen TW, Zhang XM. Functional magnetic resonance imaging in acute kidney injury: present status. Biomed Res Int 2016;2016:2027370

33. Vaidya VS, Ferguson MA, Bonventre JV. Biomarkers of acute kidney injury. Annu Rev Pharmacol Toxicol 2008;48:463-493

34. Fähling M, Seeliger E, Patzak A, Persson PB. Understanding and preventing contrast-induced acute kidney injury. Nat Rev Nephrol 2017;13:169-180 